\title{
Integration of visual and vestibulo-tactile inputs affecting apparent self-motion around the line of sight
}

\author{
ATSUKI HIGASHIYAMA \\ Ritsumeikan University, Kyoto, Japan \\ and \\ KAZUO KOGA \\ Nagoya University, Nagoya, Japan
}

\begin{abstract}
We investigated the effects of visual and vestibulo-tactile inputs on perceived self-motion. Each of 23 subjects was exposed to an optical pattern rotating around the roll axis (i.e., line of sight) while the chair, in which the subject was placed, was rotated back and forth between $\pm 70^{\circ}$ (i.e., large rolling) or between $\pm 35^{\circ}$ (i.e., small rolling) from the gravitational vertical. Each subject judged perceived velocity of self-motion under each of 16 combinations of pattern velocity and chair velocity. The main results were the following: (1) The mean estimation increased with pattern velocity, and it also increased with chair velocity; (2) to attain a constant perceived velocity of self-motion, pattern velocity was traded for chair velocity, and for the large rolling of the chair, visual inputs were more effective than vestibulotactile inputs, whereas for the small rolling, the inverse was true; (3) analyses of multiple regression, when applied to the mean estimations, showed that for both rollings of the chair, the visual component dominated over the vestibulo-tactile component, but for the small rolling of the chair, the difference in effectiveness between the two components was attenuated. We discuss these findings in terms of visual-vestibular interaction.
\end{abstract}

In this study, we investigated the relative contributions of visual and vestibulo-tactile inputs to the perception of self-motion. Suppose that a subject is exposed to a large optical pattern rotating around the line of sight (i.e., the roll axis). Probably, the subject then perceives the optical pattern to move as it does and, at the same time, has an illusory experience that his or her body is rotating in the direction opposite to the optical flow. This illusory motion of the body is referred to as visually induced selfmotion (Dunker, 1929), visual kinesthesis (Gibson, 1966), or vection (Brandt, Dichgans, \& Koenig, 1973) around the roll axis. Vection is also induced by rotating an optical pattern around a subject's vertical axis (i.e., circular vection, or yaw vection), by moving it laterally in the frontoparallel plane (i.e., linear vection), or by rotating it around the subject's horizontal axis (i.e., pitch vection).

To reveal the interaction between visual and vestibulotactile inputs, in this study, the subject was moved with a constant velocity around the roll axis while he or she saw the optical pattern rotating around the same axis. By being

We thank the reviewers, Lawrence Harris and Bob Post, for their helpful and constructive comments on the manuscript. We also thank Mark Hollins for his very critical reading of an earlier manuscript while A.H. was a visitor at the University of North Carolina at Chapel Hill. Correspondence concerning this article should be addressed to K. Koga, The Research Institute of Environmental Medicine, Nagoya University, Chikusa-ku, Nagoya464-0814,Japan (e-mail: koga@riem.nagoya-u.ac.jp). rolled with a constant velocity, a subject receives vestibular inputs from the otolith organs in the inner ears (e.g., Benson, 1990) and cutaneous inputs from the touch organs that are pressed down by the supporting structure (e.g., Lackner \& Graybiel, 1979). This real motion of body, not mediated by vision, is referred to as vestibulo-tactile motion.

In normal stationary environments in everyday life, optical flow is characterized as the reverse of a subject's body motion. For example, when the body rolls clockwise (CW), the optical flow rotates counterclockwise $(\mathrm{CCW})$ with the same velocity as the body rotation. However, in this study, we investigated not only this normal condition, but also several artificial conditions for which the combinations of optical flow and body motion are very rare in normal environments. For example, in some conditions in this study, optical flow was rotated exactly in the same direction as body motion; in other conditions, the velocity of optical flow differed from that of body motion.

By analyzing both normal and artificial conditions as mentioned above, we attempted to discover a general principle that governs perceived self-motion. When the visual inputs are in agreement or conflict with the vestibulotactile inputs, there are several possible interactions between them (for a review, see Anderson, 1986; Dichgans \& Brandt, 1977; Howard, 1982; Young, 1984). First, despite vestibulo-tactileinputs, the subject may perceive the body as rotating in the direction opposite to the rotation of the optical pattern. Second, the subject may ignore the 
visual inputs and perceive self-motion on the basis of vestibulo-tactile inputs. Such predictions would assume that the visual and the vestibulo-tactile inputs are processed separately and that the dominant inputs determine perceived self-motion. It is also very likely that the perceived self-motion may be determined through a mixture of the two types of inputs. This outcome would imply that although the visual and the vestibulo-tactile afferent inputs originate in different peripheral organs, they are integrated together at a higher processing level.

Interestingly, most studies of roll vection (Brandt, Wist, \& Dichgans, 1975; Dichgans, Held, Young, \& Brandt, 1972; Held, Dichgans, \& Bauer, 1975; Howard \& Childerson, 1994; Reason, Mayes, \& Dewhurst, 1982; Young, Oman, \& Dichgans, 1975) have focused mainly on the displacement of the visual and postural vertical, and to our knowledge, perceived self-motion has not been fully investigated. Indeed, just one study (Howard \& Childerson, 1994) has noted that a large optical pattern rotating around the roll axis often induces a paradoxical experience of (1) a stationary self that is tilted in the direction opposite to the rotating pattern and (2) a rotating self that does not accompany a continuous change of position. Nevertheless, in this study, the analysis with respect to self-motion was qualitative. The goal of the present study was, therefore, to analyze the quantitative aspects of perceived self-motion in conditions in which optical pattern motion was independent of real body motion.

\section{EXPERIMENT 1}

\section{Method}

Subjects. Twelve paid undergraduates ( 5 men and 7 women) served as the subjects; none of them had any known deficits in visual acuity or motor or vestibular functions.

Apparatus. The apparatus consisted of a rotating chair, in which the subject was placed, and a slide projector that was mounted at the top of the chair to generate an optical pattern on the inner surface of a hemisphere. Figure 1 shows the relations among the chair, the slide projector, and the screen. With the voltage servoregulation system, the chair was rotated back and forth, at a velocity of $6 \mathrm{deg} /$ sec, between a $70^{\circ}$ left tilt and a $70^{\circ}$ right tilt from the gravitational vertical. The pivot on which the body rotated was located approximately at the midpoint between the eyes. When the chair arrived at the leftmost or rightmost position, it soon began to move in the opposite direction. The time and circular acceleration that were needed for the chair to reach the constant velocity of $6 \mathrm{deg} / \mathrm{sec}$ from the stationary state were $350 \mathrm{msec}$ and $20 \mathrm{deg} / \mathrm{sec}^{2}$, respectively. In order to minimize the shifts of body that would be concomitant with the chair rotation, a very strict device was applied to the trunk, shoulder, wrist, and thigh of the subjects. The head was also stabilized with a restraining structure, so that the subject could not shake, nod, or incline his or her head. To maintain verbal communication with the experimenter, the subject did not bite a board.

The screen was placed about $150 \mathrm{~cm}$ away from the midpoint of the subject's eyes. The whole size of the screen available to the subject was $191^{\circ}$ of visual angle. On the center of the screen, a red $\mathrm{He}-\mathrm{Ne}$ laser light point was projected. The visual angle of the light point was $0.095^{\circ}$, and its intensity was reduced to less than the value of light hazard guideline (5 mW, according to ANSI Standard Z-136).

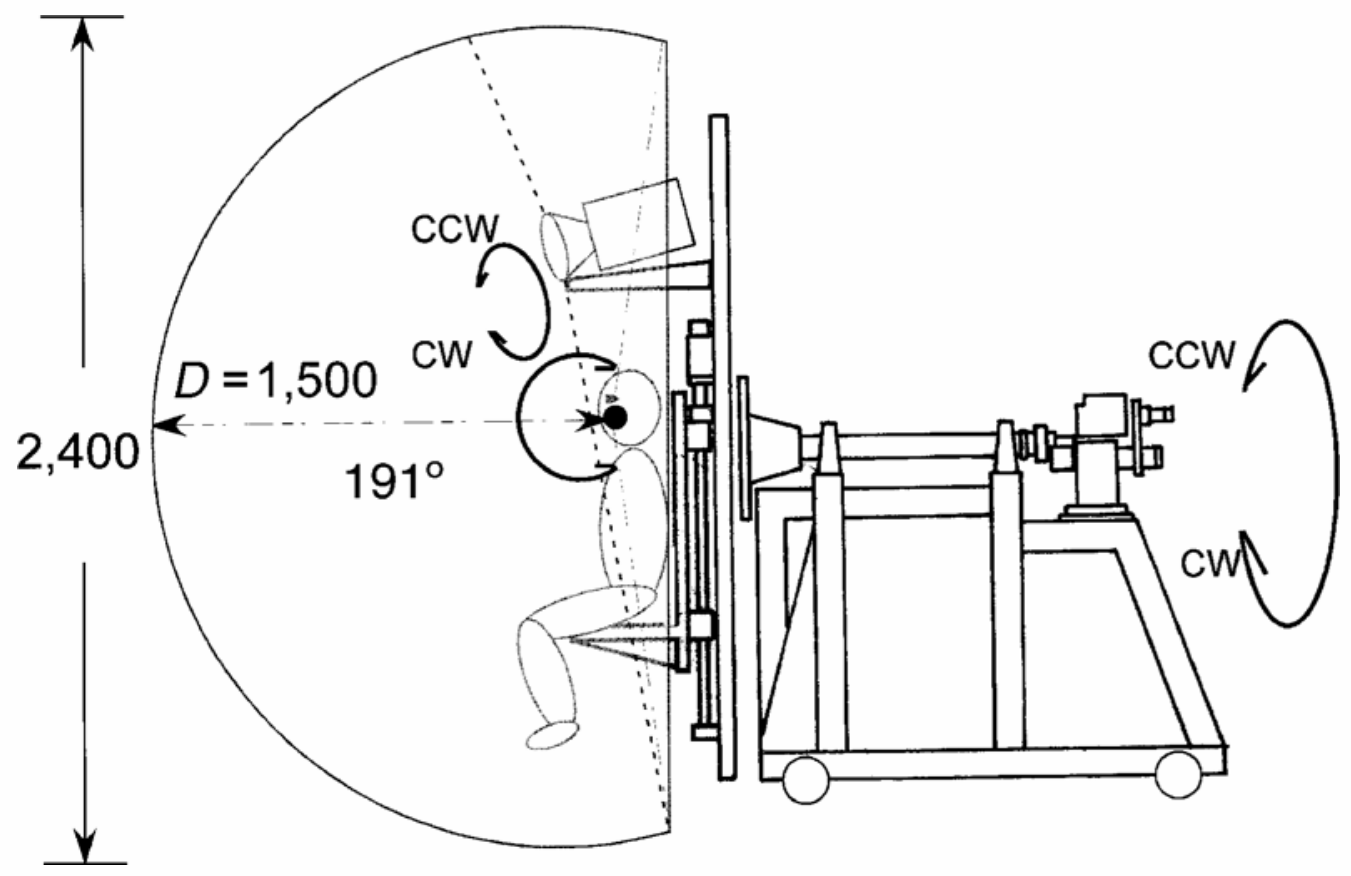

Figure 1. The side view of the apparatus in millimeters. The slide projector was placed above the subject's head and moved concomitantly with the rotation of the chair in which the subject sat. CW, clockwise; CCW, counterclockwise. 
The optical pattern, taken from a Letraset sheet (Screen-Tone No. 685), was projected on the entire screen by a slide projector set within the rotating chair. The optical pattern consisted of black irregular blobs, as is shown in Figure 2. About $30 \%$ of the entire screen was occupied by the blobs, of which the smallest was about $2 \mathrm{~cm}$ wide and $7 \mathrm{~cm}$ long, the largest was about $2-3 \mathrm{~cm}$ wide and $35 \mathrm{~cm}$ long, and the medium was about $2.0 \mathrm{~cm}$ wide and $20 \mathrm{~cm}$ long. The luminance was $2.73 \mathrm{~cd} / \mathrm{m}^{2}$ for the blobs and $3.96 \mathrm{~cd} / \mathrm{m}^{2}$ for the background.

To rotate the optical pattern CW (plus direction) or CCW (minus direction), the slide was inserted into a DC-motor-driven rotary mount that was set between the lens and the lamp in the projector. Although the projector rotated with the chair, the rotation of the slide mount in the projector, $V_{\mathrm{p}}$ (i.e., pattern velocity), was independent of the rotation of the chair, $V_{\mathrm{c}}$ (i.e., chair velocity). Also note that $V_{\mathrm{p}}$ is the velocity of the pattern that is obtained by assuming the subject's body axis (e.g., yaw axis) as the reference. To cover the entire screen with the slide pattern, a fisheye lens was fitted to the head of the lens of the projector. The center of the optical flow was at the laser light point on the screen.

Besides chair velocity and pattern velocity, we defined the composite variable $V_{\mathrm{c}}+V_{\mathrm{p}}$, which is the velocity of the pattern that would be obtained by assuming some external dimension (e.g., the gravitational vertical) as the reference. We also considered another composite variable, $V_{c}+\left(-V_{\mathrm{p}}\right)$, which is the simple sum of the two velocities that are available in the perception of self-motion. The inverted sign of $V_{\mathrm{p}}$ in this variable represents the fact that roll vection is experienced in the direction opposite to the pattern motion.

Sensitivity to acceleration and velocity. Figure 3 shows the temporal course of velocity and acceleration of the chair, which started to move from the stationary state, reached a constant velocity of $6 \mathrm{deg} / \mathrm{sec}$, and returned to the stationary state. The cupula in the semicircular canals is generally stimulated by the circular acceleration (and deceleration) of chair motion. However, when the acceleration lasts for $1 \mathrm{sec}$ or less, there is no substantial effect of acceleration on judgments of self-motion (Brown, 1966; Clark \& Stewart, 1968). Since we used a chair acceleration of 350-msec duration, the possible effects of chair acceleration on judgments of self-motion were ruled out.

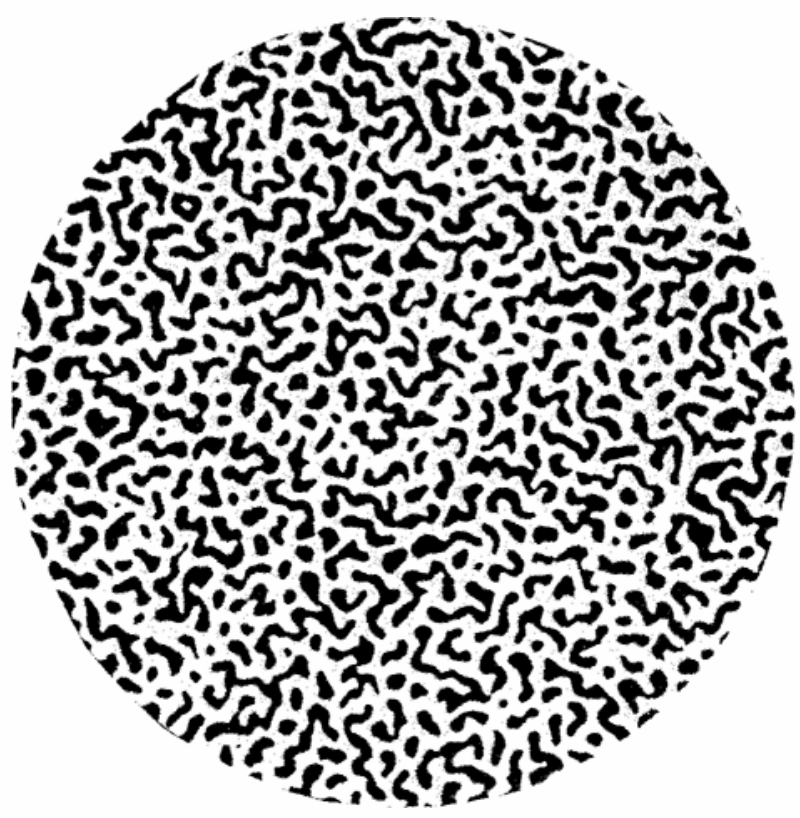

Figure 2. Optical pattern used in this study.
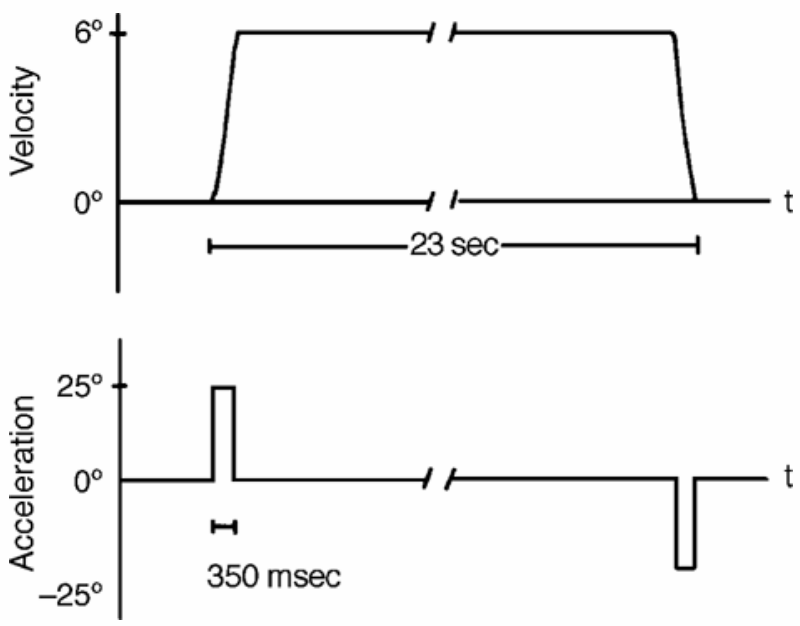

Figure 3. Velocity (deg/sec) and acceleration/deceleration $\left(\mathrm{deg} / \mathrm{sec}^{2}\right)$ of chair motion.

The otoliths in the utricle generally respond to linear acceleration, including gravity. When the body is tilted or rotated around the roll axis, the otoliths are pulled by the shearing force, the component of gravity, and they act tangentially on the macula (i.e., sensory hair cells). From the bending of the macula, we perceive how the body is tilted. From the change in the bending of the macula over time, we perceive how fast the body rolls. The chair velocity of $6 \mathrm{deg} / \mathrm{sec}$ was sufficient to perceive motion of the chair, as is shown in the Results section.

Conditions. There was a total of 16 experimental conditions, which consisted of 4 pattern conditions, 2 chair conditions, and 10 patternchair combinations. In the pattern conditions, the chair was stationary, along with the gravitational vertical, but the pattern rotated at \pm 1 or $\pm 36 \mathrm{deg} / \mathrm{sec}$. In the chair conditions, the chair rotated at $\pm 6 \mathrm{deg} / \mathrm{sec}$, and the subject's eyes were occluded to stop the visual inputs. In the pattern-chair combinations, the chair rotated at $\pm 6 \mathrm{deg} / \mathrm{sec}$, and the pattern rotated at $0, \pm 1$, or $\pm 36 \mathrm{deg} / \mathrm{sec}$. In the pattern conditions and the pattern-chair conditions, the subjects saw the optical pattern binocularly. The left half of Table 1 shows the chair velocity and the pattern velocity for each experimental condition.

Procedure. In a given trial, the subject judged the direction and velocity of self-motion. At the onset of the experiment, a stopwatch was shown to the subject. While watching the stopwatch, the subject was told that when the body was perceived to rotate in the same direction as the clock's hand, he or she should report "clockwise"; when the body was perceived to rotate in the direction opposite to it, he or she should report "counterclockwise" ; and when the body was perceived not to rotate at all, he or she should report "stationary." The subject was also asked to estimate the velocity of the selfmotion in each trial, relative to the velocity of the second hand of a clock (i.e., $6 \mathrm{deg} / \mathrm{sec}$ ). The number 10 was assigned to the velocity of the second hand. It was emphasized that the motion about which the subject should judge was his or her self-motion, not the motion of the pattern or the chair

In each of the pattern conditions, the subject was placed in the upright chair and looked at the fixation point around which the optical pattern rotated. After being exposed to the pattern for about $12 \mathrm{sec}$, the subject judged the perceived direction and velocity of selfmotion. While the subject made the judgments, the pattern continued to rotate. Immediately after the subject finished making the judgments, the rotation of the pattern was changed for the next trial. The four pattern conditions ( \pm 6 and $\pm 36 \mathrm{deg} / \mathrm{sec}$ ) were run randomly for each subject. 
Table 1

Means and Standard Deviations for Velocity Judgments for the 16 Conditions in Experiment 1

\begin{tabular}{|c|c|c|c|c|c|}
\hline \multicolumn{4}{|c|}{$\begin{array}{l}\text { Velocity } \\
\text { (deg/sec) }\end{array}$} & \multicolumn{2}{|c|}{$\begin{array}{c}\text { Estimation } \\
(\mathrm{deg} / \mathrm{sec})\end{array}$} \\
\hline$V_{\mathrm{c}}$ & $V_{\mathrm{p}}$ & $V_{\mathrm{c}}+V_{\mathrm{p}}$ & $V_{\mathrm{c}}-V_{\mathrm{p}}$ & $M$ & $S D$ \\
\hline \multicolumn{6}{|c|}{ Pattern-Chair Conditions } \\
\hline+6 & 0 & +6 & +6 & 2.3 & 3.4 \\
\hline+6 & +6 & +12 & 0 & -0.2 & 4.8 \\
\hline+6 & +36 & +42 & -30 & -10.1 & 9.0 \\
\hline+6 & -6 & 0 & +12 & 4.1 & 2.5 \\
\hline+6 & -36 & -30 & +42 & 11.4 & 5.9 \\
\hline-6 & 0 & -6 & -6 & -2.9 & 2.0 \\
\hline-6 & +6 & 0 & -12 & -4.5 & 2.6 \\
\hline-6 & +36 & +30 & -42 & -11.8 & 5.3 \\
\hline-6 & -6 & -12 & 0 & 0.5 & 3.7 \\
\hline-6 & -36 & -42 & +30 & 10.0 & 8.6 \\
\hline \multicolumn{6}{|c|}{ Pattern Conditions } \\
\hline 0 & +6 & +6 & -6 & -3.6 & 2.6 \\
\hline 0 & +36 & +36 & -36 & -12.2 & 5.9 \\
\hline 0 & -6 & -6 & +6 & 3.5 & 2.5 \\
\hline 0 & -36 & -36 & +36 & 11.6 & 5.3 \\
\hline \multicolumn{6}{|c|}{ Chair Conditions } \\
\hline+6 & no pattern & - & & 5.6 & 2.8 \\
\hline-6 & no pattern & - & & -4.8 & 2.6 \\
\hline
\end{tabular}

Note- $V_{\mathrm{c}}$, velocity of the chair; $V_{\mathrm{p}}$, velocity of the pattern, relative to the subject (or the velocity of the slide in the projector); $V_{\mathrm{c}}+V_{\mathrm{p}}$, velocity of the pattern, relative to the external stationary reference; $V_{\mathrm{c}}-V_{\mathrm{p}}$, simple sum of the two velocities available in the perception of self-motion.

In the chair conditions, the chair moved, between the two ends, at a velocity of $\pm 6 \mathrm{deg} / \mathrm{sec}$, and the blindfolded subject judged the direction and velocity of self-motion. In the $\mathrm{CW}$ condition, the chair was first at the right end and then moved toward the left end. Just when the chair was momentarily at the upright position, the experimenter prompted the subject to judge the direction and velocity of self-motion. It took about $12 \mathrm{sec}$ for the chair to travel from the end position to the upright position. While the subject judged the direction and velocity, the chair continued to move toward the left end. When the chair arrived at the left end, it soon changed the direction of motion for the next CCW trial. In the CCW condition, we repeated the same procedure as that in the $\mathrm{CW}$ condition. The $\mathrm{CW}$ and $\mathrm{CCW}$ conditions alternated with each other-that is, $\mathrm{CW}-\mathrm{CCW}-\mathrm{CW}-$ $\mathrm{CCW}$ or CCW-CW-CCW-CW.

In the pattern-chair combinations, the chair rotated, between the two ends, at a velocity of $\pm 6 \mathrm{deg} / \mathrm{sec}$, and the subject looked at the fixation point around which the optical pattern rotated. On the first trial, the chair was at one end and then moved toward the other end. When the chair was momentarily at the upright position, the subject was told to judge the direction and velocity of self-motion, and he or she was also encouraged to complete the judgments before the chair arrived at the end. When the chair arrived at the end, the direction and/or the velocity of the optical pattern and the direction of the chair were changed for the next trial.

After several practices, the subjects took part in the pattern and the chair conditions. Half the subjects first made judgments for the pattern conditions and then for the chair conditions, and the other subjects made judgments in the reverse order. Each subject made two judgments for each of the pattern and the chair conditions.

The judgments for the pattern-chair combinations followed those for the pattern and the chair conditions. There were four blocks, each of which contained the 10 different pattern-chair combinations. Thus, each subject provided four estimations for each combination.

The pattern and the chair conditions were again imposed after the subject completed the pattern-chair combinations. The procedure was the same as that in the early stage, but the order of the pattern and the chair conditions was changed: The subjects who had first done the pattern conditions in the early stage performed them after the chair conditions in the late stage, whereas the subjects who had first done the chair conditions in the early stage performed them after the pattern conditions in the late stage.

\section{Results}

We used the plus sign (+) to signify CW responses, the minus sign (-) to signify CCW responses, and the number zero to signify stationary responses. Since the subjects were required to designate the velocity of $6 \mathrm{deg} / \mathrm{sec}$ as 10 , all estimations were converted into the degree/sec equivalents by multiplying each estimation by 0.6 . Individual velocity estimations were then obtained by averaging the four estimations for each experimental condition. Table 1 shows the means and the $S D$ s of the individual velocity estimations $(n=12)$.

Pattern conditions. In the stationary chair, the subject perceived the body to rotate in the direction opposite to that of the optical pattern. For the $\mathrm{CW}$ and $\mathrm{CCW}$ patterns of $6 \mathrm{deg} / \mathrm{sec}$, the mean estimations were -3.6 and $3.5 \mathrm{deg} / \mathrm{sec}$, respectively. For the $\mathrm{CW}$ and $\mathrm{CCW}$ patterns of $36 \mathrm{deg} / \mathrm{sec}$, the mean estimations were -12.2 and $11.6 \mathrm{deg} / \mathrm{sec}$, respectively.

Chair conditions. The chair rotation of $6 \mathrm{deg} / \mathrm{sec}$ under the occluding conditions produced much larger estimation of body velocity: The mean estimations were 5.6 and $-4.8 \mathrm{deg} / \mathrm{sec}$ for the $\mathrm{CW}$ and the $\mathrm{CCW}$ chair rotations, respectively. The mean estimations for the $6-\mathrm{deg} / \mathrm{sec}$ chair rotations were significantly different from those for the 
6-deg/sec pattern rotations [for the perceived CW selfmotion, $t(11)=6.08, p<.001$; for the perceived CCW self-motion, $t(11)=2.64, p<.05$ ].

Pattern-chair combinations. Figure 4 shows the results of the pattern-chair combinations, together with those of the pattern conditions. The ordinate is the mean estimation of self-motion and the abscissa is the pattern velocity. The parameter is the chair motion. A two-way repeated measures analysis of variance (ANOVA) was performed on the data of Figure 4. The data for the stationary pattern were not included in the analysis, because we did not have any data for the stationary chair condition. The significant negative slope between pattern velocity and velocity estimation $[F(3,33)=38.72, p<.001]$ suggests that for the $\mathrm{CW}$ (or the $\mathrm{CCW}$ ) pattern rotation, the subjects were likely to perceive their bodies to rotate $\mathrm{CCW}$ (or $\mathrm{CW}$ ) and that the perceived velocity was proportional to pattern velocity.

Figure 4 also indicates that the chair motion affected the velocity estimation $[F(2,22)=3.52, p<.05]$. When compared with the estimations for the stationary chair, the estimations for the $\mathrm{CW}$ chair motion increased in the $\mathrm{CW}$ direction, and the estimations for the $\mathrm{CCW}$ chair motion increased in the $\mathrm{CCW}$ direction. The pattern $\times$ chair interaction was not significant $[F(6,66)<1.86, p>.10]$, al- though the negative slope for the stationary chair condition seems to be steeper than the slopes for the moving chair conditions.

When the chair was being rotated, the mean perceived velocity of self-motion was reduced by an exposure of the pattern to the subject. This impression is shown in Figure 5, where the estimations for the $\mathrm{CW}$ and $\mathrm{CCW}$ chair conditions were compared with those for the pattern-chair combinations in which the pattern rotated at $-6,-0$, and $6 \mathrm{deg} / \mathrm{sec}$. The ordinate is the mean estimation, and the abscissa is the chair velocity. The parameter is the pattern velocity. A two-way repeated measures ANOVA showed that the main effect of chair was significant $[F(1,11)=$ $24.78, p<.001]$, the main effect of pattern was significant $[F(3,33)=10.62, p<.001]$, and the pattern $\times$ chair interaction was significant $[F(3,33)=11.01, p<.01]$. The difference in estimation between the $\mathrm{CW}$ and the $\mathrm{CCW}$ rotations of the chair alone was $10.4 \mathrm{deg} / \mathrm{sec}$, but when the patterns rotating at $-6,-0$, and $6 \mathrm{deg} / \mathrm{sec}$ were added, the differences were reduced to $3.6,5.2$, and $4.3 \mathrm{deg} / \mathrm{sec}$, respectively.

Contours of equal-appearing velocity. Figure 4 suggests that the mean estimations were determined by some combination of the visual and the vestibulo-tactileinputs. Actually, the correlation between mean estimation and

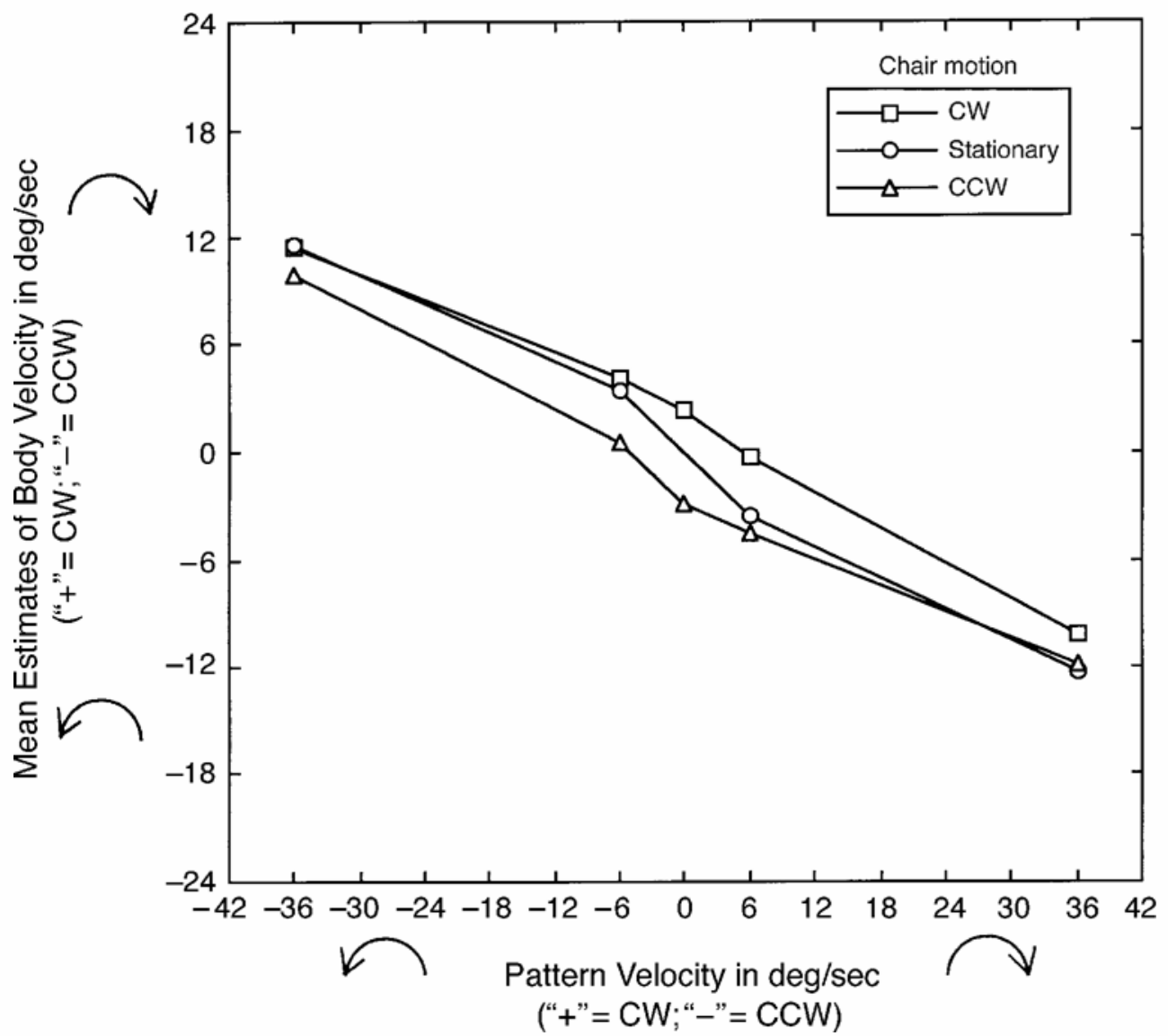

Figure 4. Mean estimations of self-motion as a function of pattern velocity, $V_{p}$, in Experiment 1 . The parameter is the chair motion, $V_{\mathrm{c}}$. CW, clockwise; $\mathrm{CCW}$, counterclockwise. 


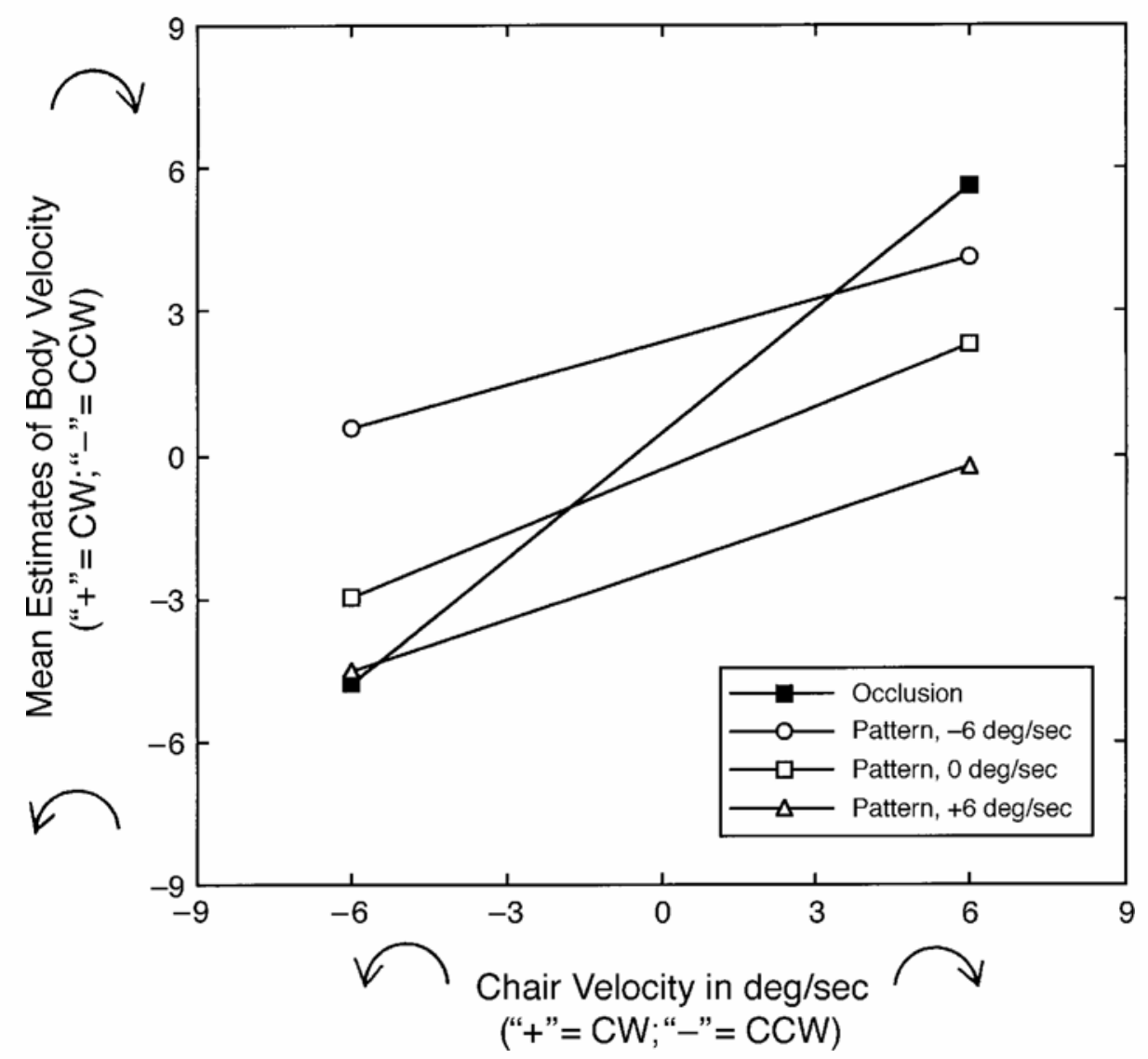

Figure 5. Mean estimations of self-motion as a function of chair velocity, $V_{c}$, in Experiment 1 . The parameter is the pattern velocity, $V_{\mathrm{p}} . \mathrm{CW}$, clockwise; $\mathrm{CCW}$, counterclockwise.

pattern velocity, $V_{\mathrm{p}}$, was -.98 , and the correlation between mean estimation and chair velocity, $V_{c}$, was .26, whereas the correlation between mean estimation and $V_{\mathrm{c}}+V_{\mathrm{p}}$ was -.92 and the correlation between mean estimation and $V_{\mathrm{c}}-V_{\mathrm{p}}$ was .99 . To examine how closely the mean estimations were related to the combination of the pattern and the chair velocities, we first constructed contours of an equal-appearing velocity from the mean estimations for the pattern-chair combinations, and second, applied the method of multiple linear regression to the mean estimations.

We attempted to determine the pattern velocity at which the body was perceived to be stationary. For each chair rotation in Figure 4, a least-square line was fitted to the mean estimation $E$ as a function of pattern velocity. It was found that for the $\mathrm{CW}$ chair rotation, $E=-.301 V_{\mathrm{p}}+1.500$ $\left(r^{2}=.99\right)$; for the CCW chair rotation, $E=-.306 V_{\mathrm{p}}-$ $1.752\left(r^{2}=.98\right)$; for the stationary chair, $E=-.338 V_{\mathrm{p}}-$ $.198\left(r^{2}=.98\right)$. The pattern velocities needed for attaining the stationary body were readily obtained by substituting $E=0$ into each of these equations. The resulting pattern velocities were $+4.98,-5.73$, and $-.59 \mathrm{deg} / \mathrm{sec}$ for the $\mathrm{CW}, \mathrm{CCW}$, and stationary chairs, respectively. This suggested that the subjects, who were in the chair rotating at the $6-\mathrm{deg} / \mathrm{sec}$ velocity, perceived their bodies to be stationary for the pattern that moved about $5.4 \mathrm{deg} / \mathrm{sec}$ in the same direction as the chair [i.e., $(4.98+5.73) / 2$ ].

With these equations, we also obtained the pairs of pattern and chair velocities for the other perceived velocities of self-motion (i.e., $E= \pm 6 \mathrm{deg} / \mathrm{sec}$ and $E= \pm 12 \mathrm{deg}$ / $\mathrm{sec})$. Figure 6 shows the results, where the ordinate is the pattern velocity and the abscissa is the chair velocity, with the perceived velocity as the parameter.

Note that the contours of the equal-appearing velocity represent how the visual and vestibulo-tactile inputs are related to maintain a constant perceived velocity of selfmotion. If the slope of the contours is parallel to the abscissa (zero slope), the perceived self-motion is exclusively determined by pattern velocity. If the slope of the contours is parallel to the ordinate (infinitely large slope), the perceived self-motion is exclusively determined by chair velocity. If the slope of the contours exactly equals unity, pattern velocity contributes as greatly as chair velocity. In short, the steeper the slope, the more the contribution of the vestibulo-tactile inputs.

Clearly, Figure 6 indicates that both pattern and chair velocities influenced the perceived velocity of self-motion. The least-square lines fitted to the five contours were $V_{\mathrm{p}}=.95 V_{\mathrm{c}}+37.75\left(r^{2}=.84\right), V_{\mathrm{p}}=.92 V_{\mathrm{c}}+18.66\left(r^{2}=\right.$ $.95), V_{\mathrm{p}}=.89 V_{\mathrm{c}}-.44\left(r^{2}=.99\right), V_{\mathrm{p}}=.87 V_{\mathrm{c}}-19.54$ 
$\left(r^{2}=.96\right)$, and $V_{\mathrm{p}}=.84 V_{\mathrm{c}}-38.64\left(r^{2}=.84\right)$ for " -12, , “-6," " 0 ," "6," and " $12 \mathrm{deg} / \mathrm{sec}$," respectively. The slope averaged .89 , suggesting that pattern velocity was more weighted than chair velocity.

Multiple linear regression. The mean estimations for the pattern-chair combinations, in which both the pattern and the chair moved, say, at $6 \mathrm{deg} / \mathrm{sec}$, were somewhere between the mean estimations for the $6-\mathrm{deg} / \mathrm{sec}$ pattern condition and those for the $6-\mathrm{deg} / \mathrm{sec}$ chair conditions. The mean estimation for the $\mathrm{CW}$ pattern rotation under the CCW chair motion $(-4.5 \mathrm{deg} / \mathrm{sec})$ was between the mean estimation for the $\mathrm{CW}$ pattern condition $(-3.6 \mathrm{deg} /$ $\mathrm{sec}$ ) and the mean estimation for the $\mathrm{CCW}$ chair condition $(-4.8 \mathrm{deg} / \mathrm{sec})$. Likewise, the mean estimation for the $\mathrm{CCW}$ pattern rotation under the $\mathrm{CW}$ chair motion ( $4.1 \mathrm{deg} /$ $\mathrm{sec}$ ) was between the mean estimation for the $\mathrm{CCW}$ pat- tern condition $(3.5 \mathrm{deg} / \mathrm{sec})$ and the mean estimation for the $\mathrm{CW}$ chair condition $(5.6 \mathrm{deg} / \mathrm{sec})$.

Not only the pattern-chair combinations in which both the pattern and the chair moved at $6 \mathrm{deg} / \mathrm{sec}$, but also other combinations may be able to be predicted from the estimations for the pattern and the chair conditions. For simplicity, when the mean estimations for the patternchair combinations $(E)$ were represented as a multiple linear function of the estimation for the pattern $(P)$ and the chair $(C)$ conditions, we obtained the function $E=.89 P$ $+.31 C$, with an adjusted squared coefficient of multiple regression $R^{2}$ of .98 . For the variable $P$, the standard coefficient $\beta$ was .97 $[t(7)=21.3, p<.001]$, and for the variable $C, \beta$ was $.22[t(7)=4.9, p<.01]$. Figure 7 shows how well the estimations for the pattern-chair combinations are predicted as a linear sum of the two sensory com-

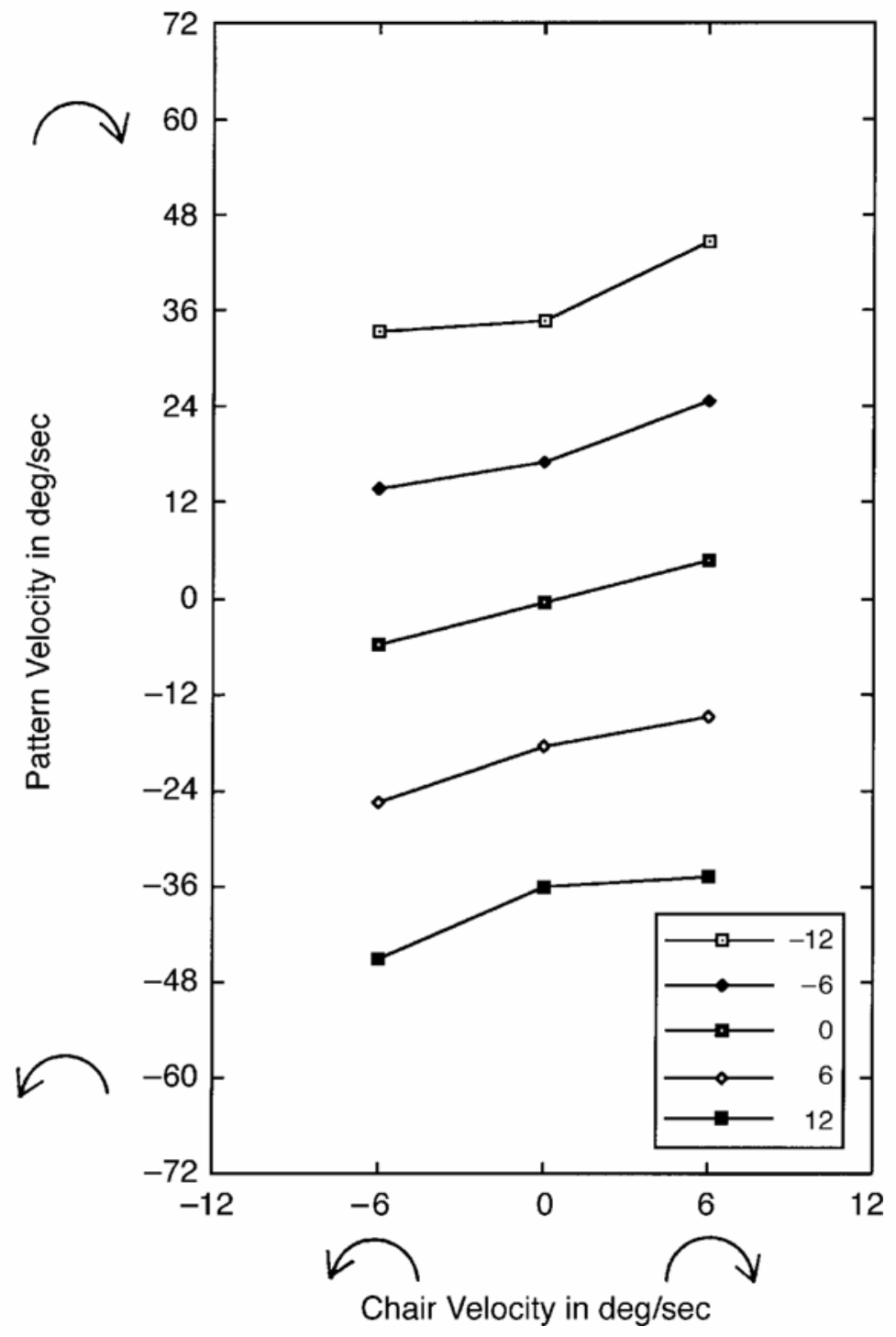

Figure 6. Relations between pattern velocity and chair velocity that were required to maintain perceived body motions of " -12, , " $-6, "$ " 0 ," " 6 ," and “12" deg/sec in Experiment 1. 
ponents. We also represented the mean estimations as a multiple linear function of $V_{\mathrm{p}}$ and $V_{\mathrm{c}}$. The equation obtained was $E=-.30 V_{\mathrm{p}}+.27 V_{\mathrm{c}}\left[R^{2}=.98 ; \beta\right.$ for $V_{\mathrm{p}}$ was $-.97, t(7)=23.7, p<.001$, and $\beta$ for $V_{\mathrm{c}}$ was $.23, t(7)=$ $5.5, p<.01]$. Since the $\beta$ values in these analyses were very similar to one another, in the next experiment, we applied only the equation that assumes $P$ and $C$ as independent variables.

\section{EXPERIMENT 2}

In Experiment 1, all the slopes for the contours of equalappearing velocity were less than unity, and the standard coefficient for the visual component was larger than that for the vestibulo-tactile component. These findings suggest that the visual inputs were much more weighted than were the vestibulo-tactile inputs. In Experiment 2, we again examined the effects of visual and vestibulo-tactile inputs on perceived self-motion by halving the path length of the chair rotation and by halving the velocities of both the pattern and the chair. In short, the chair rotated slowly over a shorter range, and the pattern also rotated slowly. The subject then judged perceived self-motion at the moment of the chair's being upright.

We assumed that the effects of vestibulo-tactile inputs on perceived self-motion would be enhanced in Experi- ment 2. This assumption was made from previous studies (Graybiel \& Patterson, 1955; Mittelstaedt, 1999; Parker, Wood, Gulledge, \& Goodrich, 1979; Quix, 1925; Schöne, 1964; Schöne \& Mortag, 1968; Schöne \& Wade, 1971; Udo de Haes, 1970; Young et al., 1975) that have shown that the otoliths are probably sensitive to small body tilts near the gravitational vertical. Note that the duration for which the subjects were exposed to the small body tilts in Experiment 2 was exactly twice as long as the duration for which they were exposed to the same body tilts in Experiment 1. This means that in Experiment 2, the sensitive neural responses of the otoliths would be transmitted to the central nervous system more.

One could make another assumption that if the subjects based their judgments on the neural responses at the moment of the chair's being upright, the relative effects of visual and vestibulo-tactile inputs would be the same in both experiments. However, this assumption is not logically valid, because the momentary shearing force acting tangentially on the macula provides us with information as to how the body is tilted, but not how fast it is moved. To perceive velocity of self-motion, we need a change of the shearing force (or thereby, a change of the bending of the macula) over time. In this study, the velocity judgments were affected by whole responses of the otoliths that were generated during the chair rotation, even

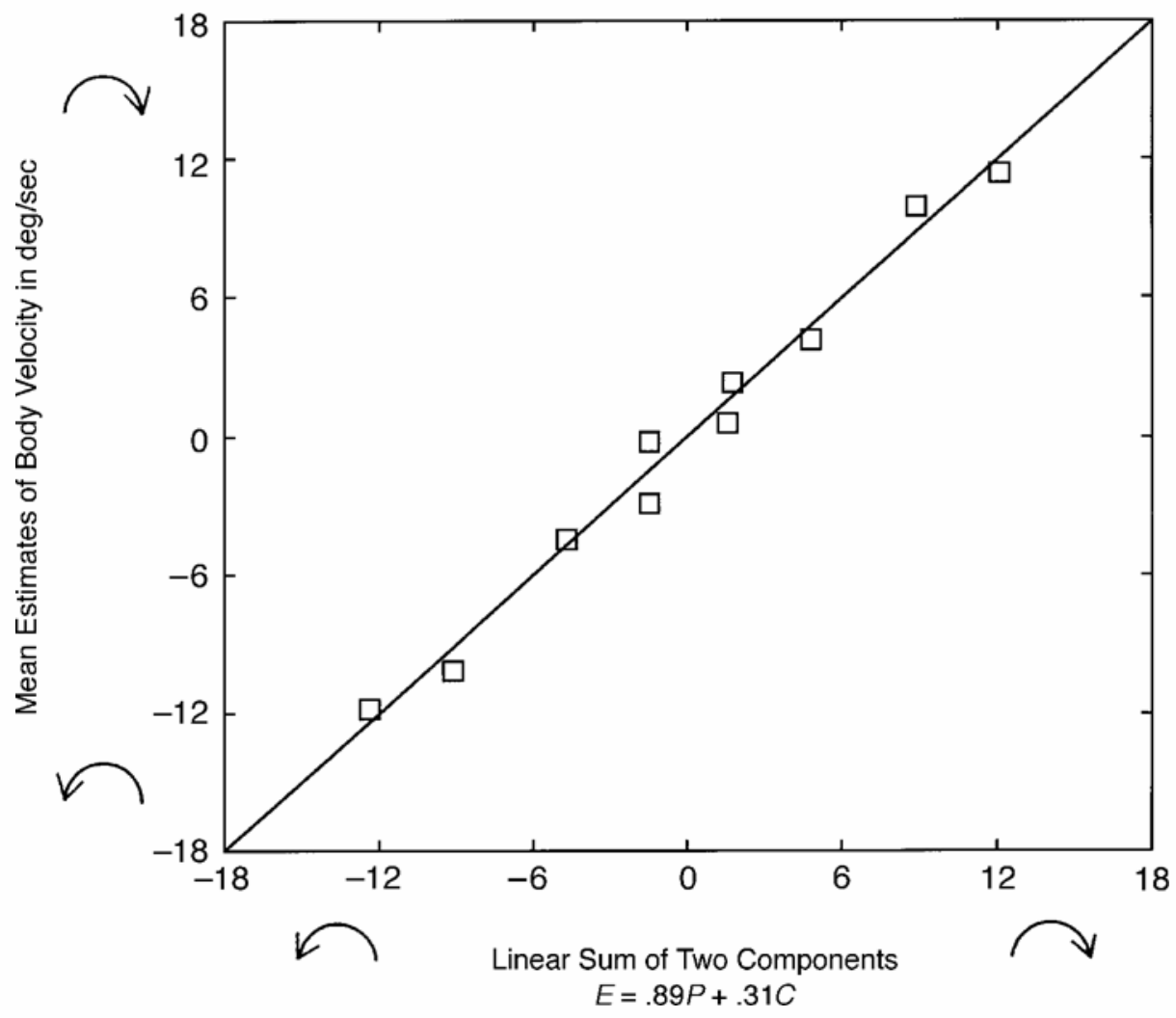

Figure 7. Relations between the mean estimations in the pattern-chair conditions and the linear sums of the estimations for the pattern and the chair conditions in Experiment 1. 
though the subjects judged at the moment of the body's being upright.

\section{Method}

Subjects. Eleven paid undergraduates (4 men and 7 women) were newly recruited as subjects; none of them had any known deficits in visual acuity or motor or vestibular functions.

Apparatus, Stimuli, and Procedure. We used the same apparatus as that in Experiment 1. The velocities of the pattern and the chair were just half as fast as those in Experiment 1: The pattern velocities were $0, \pm 3$, and $\pm 18 \mathrm{deg} / \mathrm{sec}$, whereas the chair velocity was $\pm 3 \mathrm{deg} / \mathrm{sec}$. In addition, the chair rotated between $+35^{\circ}$ and $-35^{\circ}$ from the gravitational vertical. It follows that the time that was needed for the chair to travel from one end to the other was exactly the same as that in Experiment 1. The left half of Table 2 shows the experimental conditions in Experiment 2. The procedure in Experiment 2 was the same as that in Experiment 1.

\section{Results}

Individual velocities were obtained by averaging the four estimations in each experimental condition. Table 2 shows the means and the $S D$ s of the individual velocities $(n=$ 11). The comparisons between the pattern and the chair conditions revealed that the mean estimation for the $\mathrm{CW}$ chair rotation was significantly different from that for the -3-deg/sec pattern rotation $[4.1 \mathrm{vs} .0 .7 \mathrm{deg} / \mathrm{sec} ; t(10)=$ $7.00, p<.001]$ and that the mean estimation for the CCW chair rotation was significantly different from that for the $3-\mathrm{deg} / \mathrm{sec}$ pattern rotation $[-3.7 \mathrm{vs} .-1.0 \mathrm{deg} / \mathrm{sec}, t(10)=$ $7.45, p<.001]$.

Figure 8 shows the results of the pattern-chair combinations, together with those of the pattern conditions. The main effect of pattern $[F(3,30)=23.71, p<.001]$ and the main effect of chair $[F(2,20)=8.97, p<.01]$ were significant. The pattern $\times$ chair interaction was not significant $[F(6,60)=0.46, p>.10]$.

In Figure 9, the estimations for the occluding chair are compared with those for the pattern-chair combinations in which the pattern moved at $-3,0$, and $3 \mathrm{deg} / \mathrm{sec}$. The main effect of chair was significant $[F(1,10)=32.16, p<$ $.001]$; the main effect of pattern was not significant, but the pattern $\times$ chair interaction was significant $[F(3,30)=$ $20.62, p<.001$ ], suggesting that the perceived velocity of self-motion was reduced by the exposure of the pattern to the subject.

To obtain contours of equal-appearing velocity, we fitted least-square lines to the mean estimations in Figure 8. For the CW, CCW, and stationary chairs, $E=-.251 V_{\mathrm{p}}+$ $2.498\left(r^{2}=.98\right), E=-.238 V_{\mathrm{p}}-2.167\left(r^{2}=.99\right)$, and $E=-.289 V_{\mathrm{p}}+.157\left(r^{2}=.99\right)$, respectively. From these equations, we obtained the pairs of pattern and chair velocities that were needed for maintaining constant perceived velocities of self-motion. The results are shown in Figure 10. The least-square lines fitted to these contours were $V_{\mathrm{p}}=2.96 V_{\mathrm{c}}+23.76\left(r^{2}=.95\right), V_{\mathrm{p}}=3.07 V_{\mathrm{c}}+12.11$ $\left(r^{2}=.99\right), V_{\mathrm{p}}=3.18 V_{\mathrm{c}}+.46\left(r^{2}=.99\right), V_{\mathrm{p}}=3.29 V_{\mathrm{c}}-$ $11.18\left(r^{2}=.99\right)$, and $V_{\mathrm{p}}=3.39 V_{\mathrm{c}}-22.83\left(r^{2}=.95\right)$ for “-6," “-3," " 0 ," " 3 ," and " $6 \mathrm{deg} / \mathrm{sec}$," respectively. The slope averaged 3.18 , suggesting that chair velocity was more weighted than was pattern velocity.

The mean estimations for the pattern-chair combinations were represented as a multiple linear function of the

Table 2

Means and Standard Deviations for Velocity Judgments for the 16 Conditions in Experiment 2

\begin{tabular}{|c|c|c|c|c|c|}
\hline \multicolumn{4}{|c|}{$\begin{array}{c}\text { Velocity } \\
(\text { deg/sec) }\end{array}$} & \multicolumn{2}{|c|}{$\begin{array}{c}\text { Estimation } \\
(\mathrm{deg} / \mathrm{sec})\end{array}$} \\
\hline$V_{\mathrm{c}}$ & $V_{\mathrm{p}}$ & $V_{\mathrm{c}}+V_{\mathrm{p}}$ & $V_{\mathrm{c}}-V_{\mathrm{p}}$ & $M$ & $S D$ \\
\hline \multicolumn{6}{|c|}{ Pattern-Chair Conditions } \\
\hline+3 & 0 & +3 & +3 & 1.9 & 1.5 \\
\hline+3 & +3 & +6 & 0 & 1.6 & 2.7 \\
\hline+3 & +18 & +21 & -15 & -1.7 & 6.8 \\
\hline+3 & -3 & 0 & +6 & 2.5 & 1.5 \\
\hline+3 & -18 & -15 & +21 & 7.5 & 2.6 \\
\hline-3 & 0 & -3 & -3 & -1.8 & 1.7 \\
\hline-3 & +3 & 0 & -6 & -2.5 & 1.5 \\
\hline-3 & +18 & +15 & -21 & -6.7 & 2.4 \\
\hline-3 & -3 & -6 & 0 & -1.5 & 2.6 \\
\hline-3 & -18 & -21 & 15 & 2.0 & 7.7 \\
\hline \multicolumn{6}{|c|}{ Pattern Conditions } \\
\hline 0 & +3 & +3 & -3 & -1.0 & 0.7 \\
\hline 0 & +18 & +18 & -18 & -4.7 & 2.7 \\
\hline 0 & -3 & -3 & +3 & 0.7 & 0.6 \\
\hline 0 & -18 & -18 & +18 & 5.7 & 4.3 \\
\hline \multicolumn{6}{|c|}{ Chair Conditions } \\
\hline+3 & no pattern & - & - & 4.1 & 1.5 \\
\hline-3 & no pattern & - & - & -3.7 & 1.1 \\
\hline
\end{tabular}

Note $-V_{\mathrm{c}}$, velocity of the chair; $V_{\mathrm{p}}$, velocity of the pattern, relative to the subject (or the velocity of the slide in the projector); $V_{\mathrm{c}}+V_{\mathrm{p}}$, velocity of the pattern, relative to the external stationary reference; $V_{\mathrm{c}}-V_{\mathrm{p}}$, simple sum of the two velocities available in the perception of self-motion. 


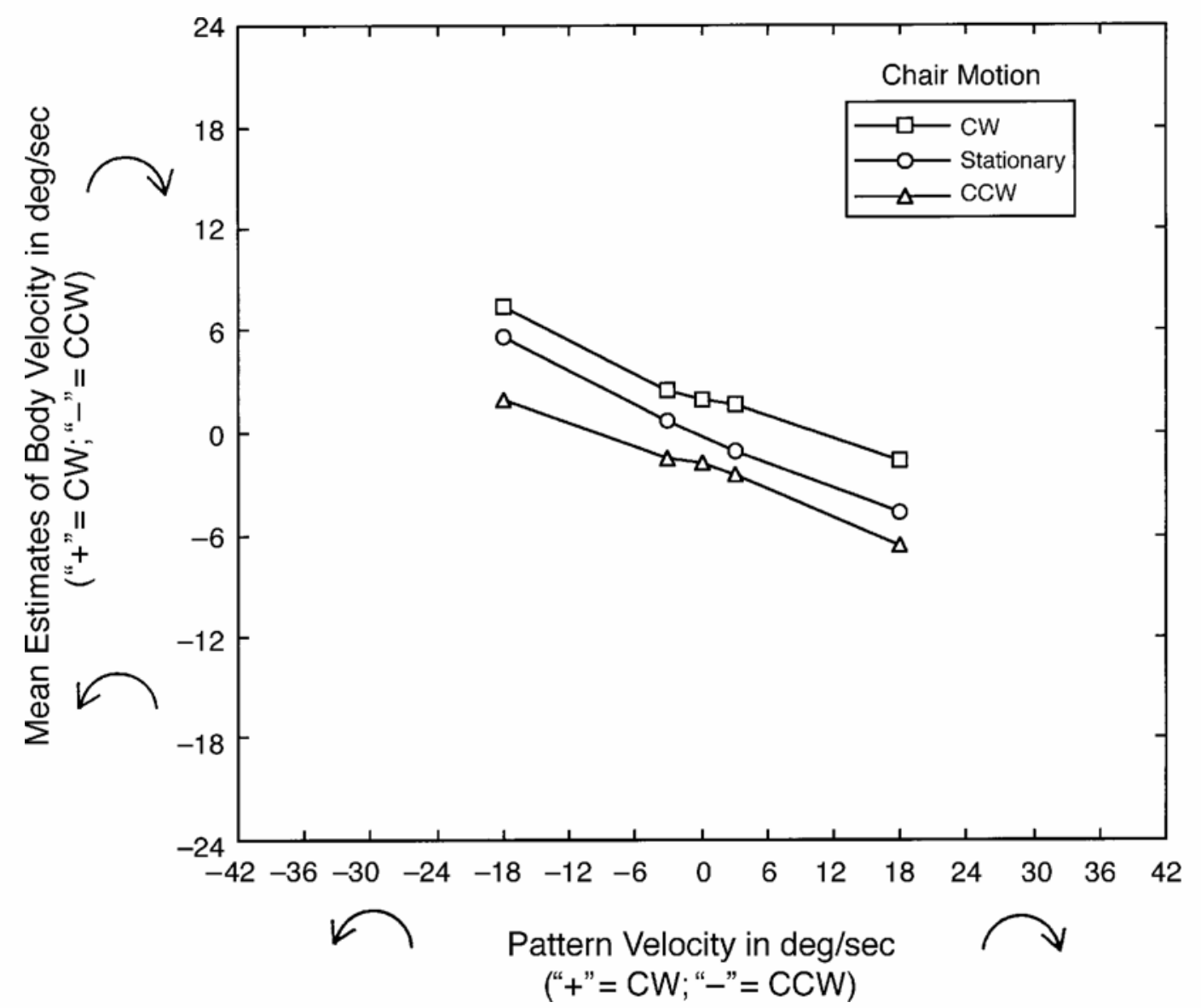

Figure 8. Mean estimations of self-motion as a function of pattern velocity, $V_{p}$, in Experiment 2. The parameter is the chair motion, $V_{\mathrm{c}} \cdot \mathrm{CW}$, clockwise; $\mathrm{CCW}$, counterclockwise.

estimations for the pattern and the chair conditions. The function that fitted best to the data was $E=.84 P+.58 C$, with an adjusted $R^{2}$ of .99 , and the estimated coefficients of the predictors were significant [for the variable $P, \beta=$ $.78, t(7)=19.16, p<.001$; for the variable $C, \beta=.62$, $t(7)=15.19, p<.001]$. The $\beta$ value for the visual component was larger than that for the vestibulo-tactile component. Figure 11 shows how well the estimations for the pattern-chair combinations were explained by the multiple linear regression.

\section{COMPARISON OF EXPERIMENTS 1 AND 2}

In Figure 12, the results for the pattern and the chair conditions in Experiments 1 and 2 are compared. Clearly, the velocity estimation $E$ for the pattern conditions increased as a linear function of pattern velocity. The least-square line that was fitted to these data points through the origin was $E=-.33 V_{\mathrm{p}}$. This equation suggests that the velocity of roll vection that was produced by the rotating pattern was about a third as fast as the pattern velocity. Similarly, the velocity estimation for the chair conditions increased as a function of chair velocity (i.e., $E=.95 V_{\mathrm{c}}$ ). Clearly, the perceived velocity of self-motion that was produced by the chair motion approximated the objective velocity of the chair.

Figures 4 and 8 suggest that pattern velocity and chair velocity additionally contributed to perceived velocity of self-motion, because in both experiments, the main effect of pattern and the main effect of chair were significant but the pattern $\times$ chair interaction was not significant. Likewise, Figures 6 and 10 suggest that both pattern velocity and chair velocity influenced the perceived velocity, because the slopes for the contours of equal-appearing velocity were consistently larger than zero in both experiments.

Moreover, the contours of equal-appearing velocity in Figures 6 and 10 help us to specify the relation between the visual and the vestibulo-tactile inputs. The slopes for the contours were less than unity in Experiment 1 but were more than unity in Experiment 2. This finding suggests that in Experiment 1, the visual inputs dominated over the vestibulo-tactile inputs, whereas in Experiment 2, the vestibulo-tactile inputs prevailed.

The analyses of the multiple linear regression suggested that the mean estimations for the pattern-chair combina- 
tions were successfully predicted by the linear combination of the estimations for the pattern and the chair conditions and that, in Experiment 1, the visual component $(\beta=$ .97) was more weighted than was the vestibulo-tactile component $(\beta=.22)$ but, in Experiment 2, although the weight of the visual component (.78) was still larger than that of the vestibulo-tactile component (.62), the $\beta$ values for the two components were much closer.

From these analyses, it may be concluded that the visual component generally had more of an effect on the perceived velocity of self-motion than did the vestibulo-tactile component, but that the availability of the vestibulo-tactile component was enhanced in Experiment 2.

\section{GENERAL DISCUSSION}

\section{Pattern and Chair Conditions}

Figure 12 indicates that when the pattern and the chair were separately moved at the same velocity (e.g., $6 \mathrm{deg} /$ $\mathrm{sec})$, the perceived self-motion induced by the pattern alone was judged to be slower than that produced by the chair alone. Harris, Jenkin, and Zikovitz (2000) obtained a similar finding in a study of linear self-motion. They gave the subject constant accelerated motion by present- ing optical flow in a virtual real pattern and/or by moving the subject passively in the dark. The perceived distance of motion induced by optical flow was found to be much smaller than that generated by the real motion of the subject's body.

How could we explain this difference in perceived selfmotion between the pattern and the chair conditions? One possible explanation is that the visually induced self-motion was not in a steady state in this study. According to previous studies of linear or circular vection (Berthoz, Pavard, \& Young, 1975; Brandt et al., 1973; Wong \& Frost, 1978), when a subject is suddenly exposed to a horizontally moving visual pattern, he or she initially experiences movement of the visual field. Within 1-5 sec, perceived body acceleration opposite to the direction of the visual field motion begins, during which the visual field seems to move progressively slower. Within 10-30 sec after stimulus onset, only self-motion is perceived, and the visual field seems to be stationary. The steady-state velocity of the induced selfrotation increases at the same rate as an increase of stimulus velocity up to $90-120 \mathrm{deg} / \mathrm{sec}$ (Kennedy, Hettinger, Harm, Ordy, \& Dunlap, 1996; Wong \& Frost, 1978).

An alternative explanation is a perceptual conflict that is produced when roll vection is experienced (Dichgans

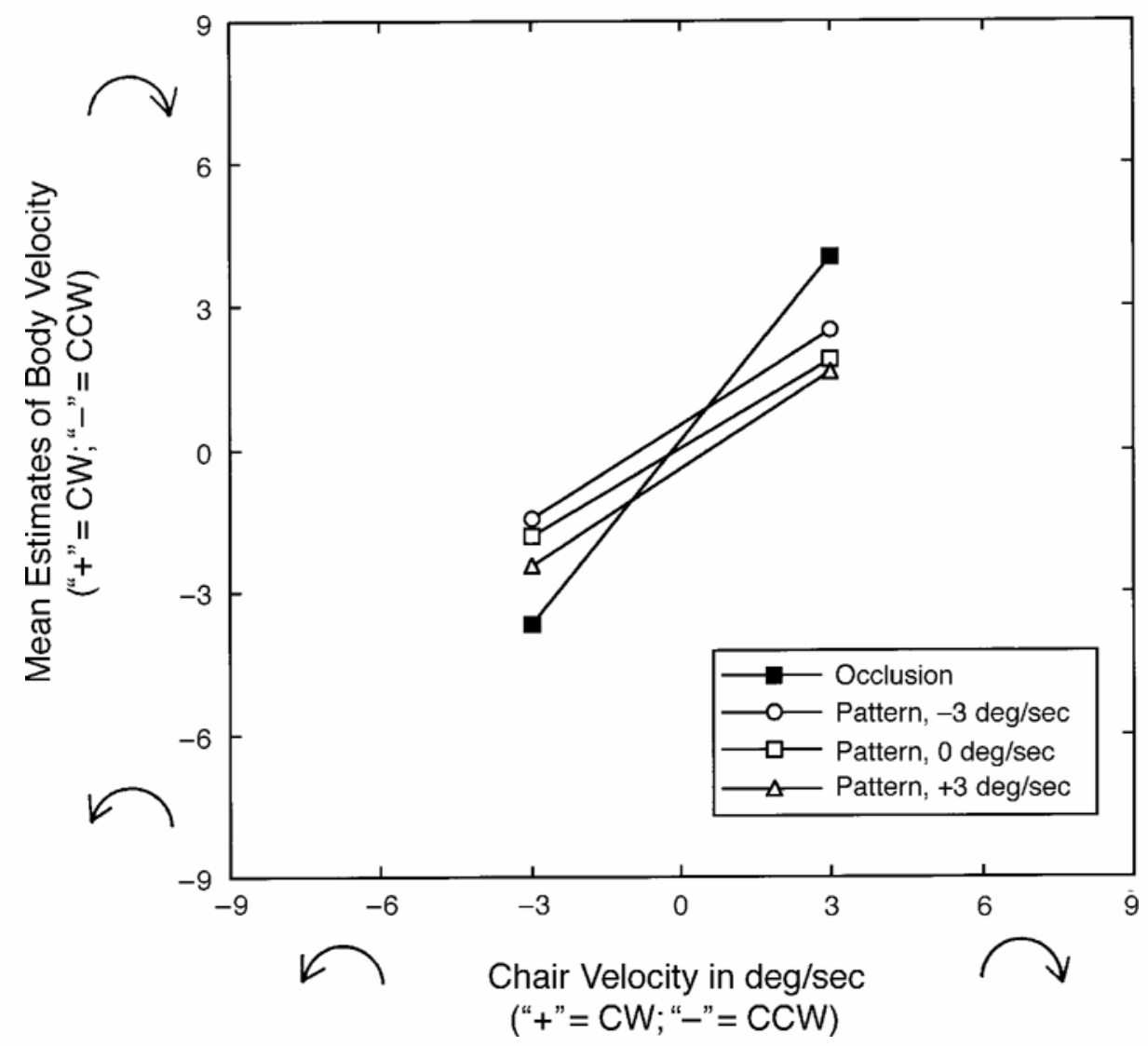

Figure 9. Mean estimations of self-motion as a function of chair velocity, $V_{c}$, in Experiment 2. The parameter is the pattern velocity, $V_{\mathrm{p}} . \mathrm{CW}$, clockwise; $\mathrm{CCW}$, counterclockwise. 


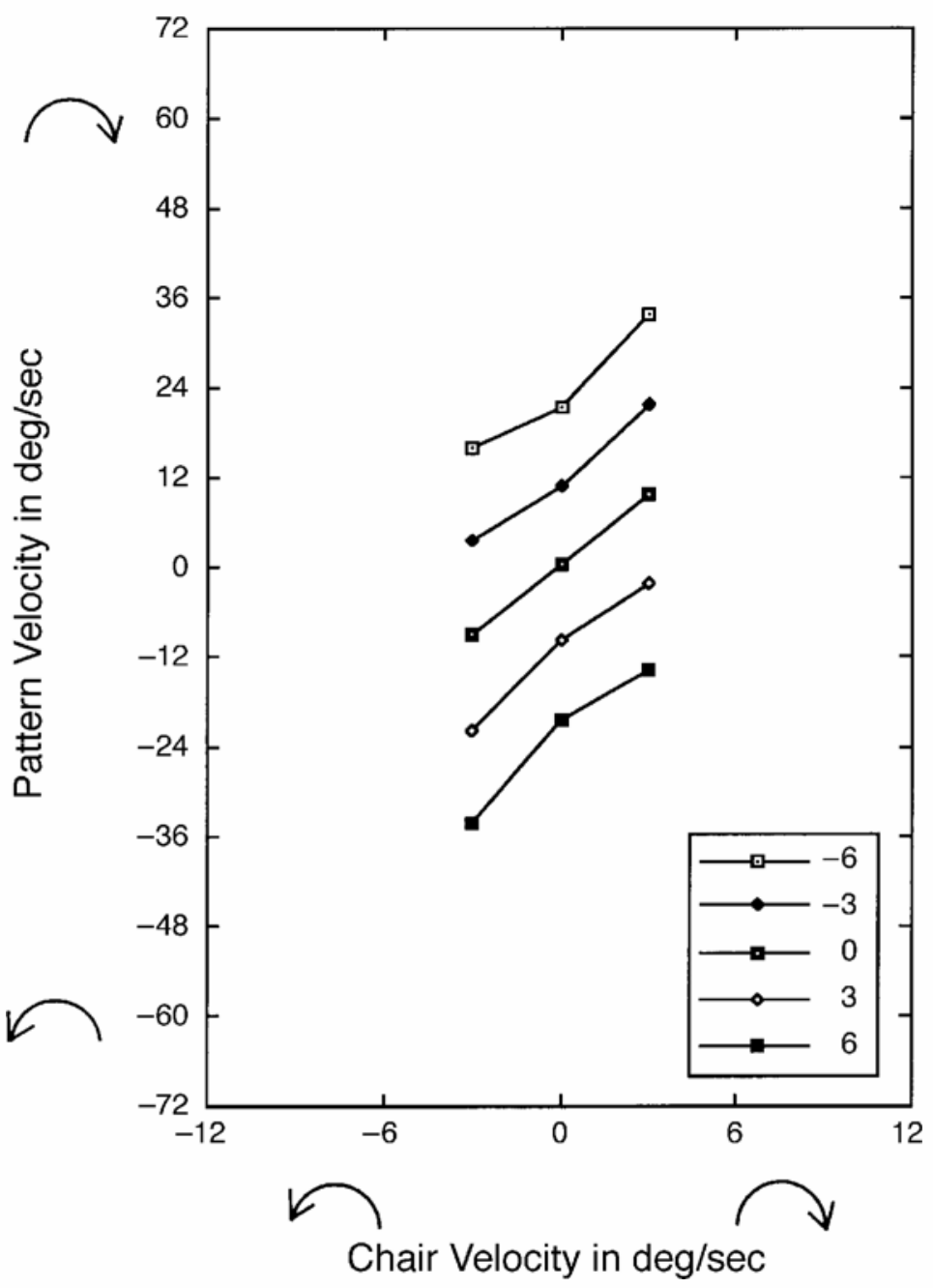

Figure 10. Relations between pattern velocity and chair velocity that were required to maintain perceived body motions of " -6, , " -3, , " 0 ," " 3 ," and " 6 " $\mathrm{deg} / \mathrm{sec}$ in Experiment 2.

\& Brandt, 1977; Howard, 1982). When the pattern rotates around the roll axis of a standing subject, the body is perceived to be tilted or rotated, but the afferent inputs from the otoliths and cutaneous receptors suggest that the body is standing stationarily. Accordingly, in roll vection, the visual inputs are in conflict with the vestibular (Dichgans \& Brandt, 1977) and tactile inputs (Brandt, Büchele, $\&$ Arnold, 1977), so that the visually induced self-motion may be attenuated.

Several studies have indeed suggested that vection is enhanced in weightlessness when visual inputs are not in conflict with other sensory inputs. Young, Crites, and Oman (1983) and Young, Oman, Watt, Money, and Lichtenberg (1984) showed that the latency to onset of roll vection was shortest in a weightless parabolic flight situation and that a longer latency to onset of vection was caused by increasing gravitational acceleration and by adding tactile cues to the subject's feet. Young and Shelhamer (1990) demonstrated that the maximum intensity and total saturation time of vection were larger in weightlessness than on the earth.

However, several outcomes of the present study do not seem to support the conflict hypothesis. Figures 5 and 9 show that the slope for the chair condition was steeper than the slope for the pattern-chair combination in which the stationary or rotating pattern was added. This means that the perceived velocity of self-motion produced by chair motion alone was reduced by the exposure of the pattern to the subject. This reduction of perceived velocity took place even for the physically stationary pattern (i.e., $V_{\mathrm{p}}=0$ ), for which the visual inputs were not in conflict with the vestibulo-tactile inputs.

\section{Pattern-Chair Combinations}

The main results of the pattern-chair combinations can be summarized as follows: (1) When the subject in a ro- 
tating chair was exposed to the optical pattern rotating around the roll axis, the perceived velocity of self-motion was affected by both pattern and chair velocities; (2) the visual inputs were generally much more effective than the vestibulo-tactile inputs; but (3) availability of vestibulotactile inputs was enhanced when the body was rolling slowly near the gravitational vertical.

Several studies have attempted to clarify the nature of the visual and vestibular inputs in the perception of selfmotion. Klinke and Schmidt (1970), Lackner and Teixeira (1977), and Teixeira and Lackner (1979) showed that (1) if a subject moved his or her head while gazing at a visual pattern rotating around a yaw axis, circular vection was suppressed, (2) the time to onset of circular vection was shorter for a stationary head than for a moving head, and (3) the visual stimulus velocity that was needed for circular vection to take place was slower for a stationary head than for a moving head. These results suggest that circular vection is weakened by motion of the head. Van der Steen and Brockhoff (2000) also demonstrated that steady-state velocity of vection was impaired by inertial body motion.

There is evidence that visual inputs, rather than vestibulo-tactileinputs, may be the dominant source of information for determining self-motion. By translating the wall and ceiling of a suspended room back and forth and placing a subject on a trolley inside the room, Lishman and Lee (1973) examined the effects of visual and vestibulotactile inputs on perceived self-motion. In one condition, the room was moved with or against the subject's motion. They found that the visual inputs alone could produce a strong body motion and usually dominated the conflicting vestibulo-tactile inputs. Berthoz et al. (1975) found that when a horizontal linear motion of a visual field was combined with a passive motion of a subject on a cart, body motion was mostly perceived to be in the same direction as the linear vection.

In agreement with the results of our study, however, some evidence (Carpenter-Smith, Futamura, \& Parker, 1995; Harris et al., 2000; Huang \& Young, 1987; Melcher \& Henn, 1981; Ohmi, 1996; Young, Dichgans, Murphy, \& Brandt, 1973) suggests that perceived self-motion is influenced by both visual and vestibulo-tactile inputs. Young et al. (1973) studied the perception of angular acceleration of the body. The subject was placed in a trainer that rotated at various angular accelerations and gazed at a visual pattern rotating at a constant velocity around the vertical body axis. For the trainer that moved in the same direction as the circular vection (i.e., the same condition), the magnitude of perceived body motion was enhanced, but for the trainer that was moved in the direction opposite

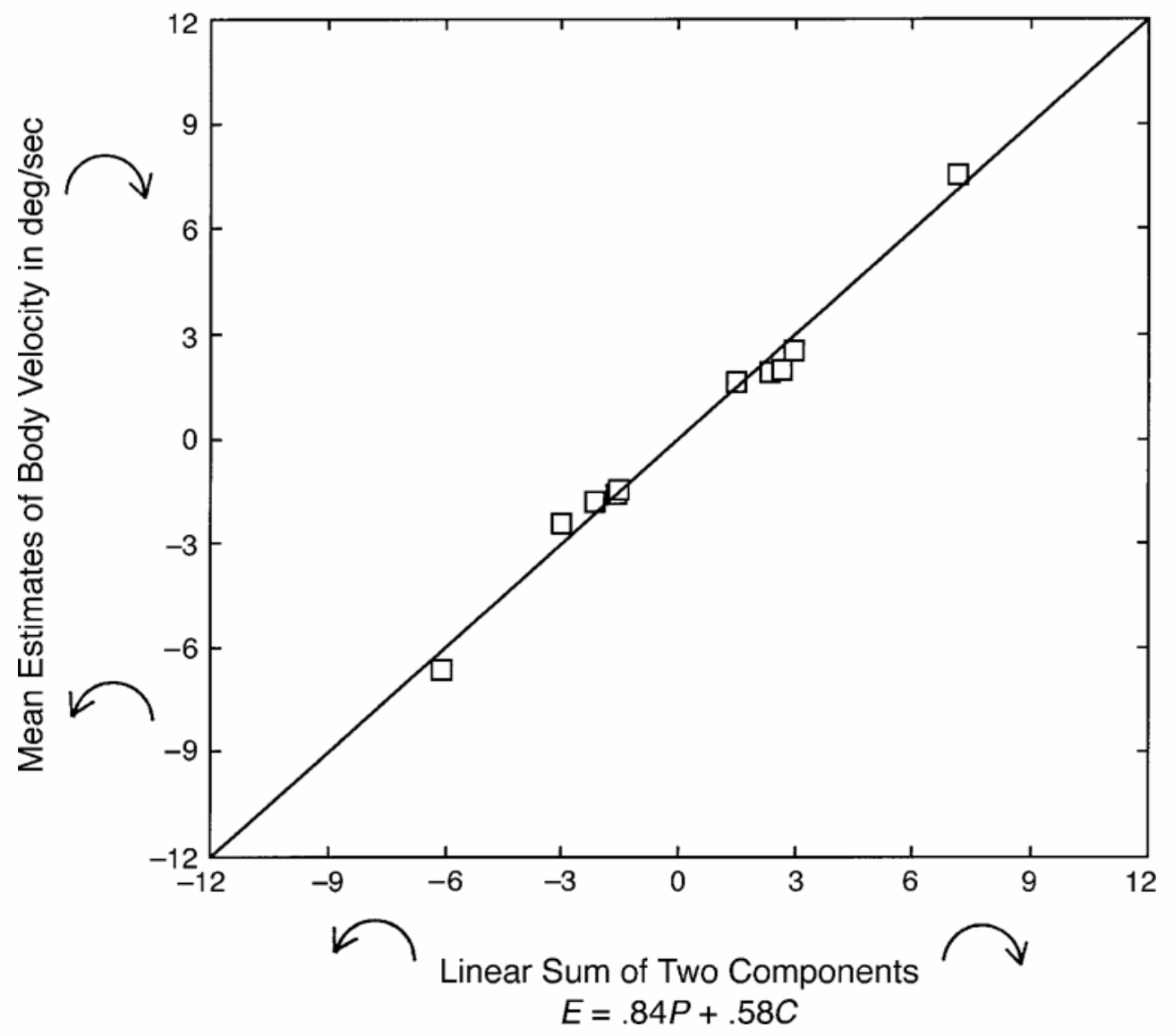

Figure 11. Relation between the mean estimations in the pattern-chair conditions and the linear sums of the estimations for the pattern and the chair conditions in Experiment 2. 


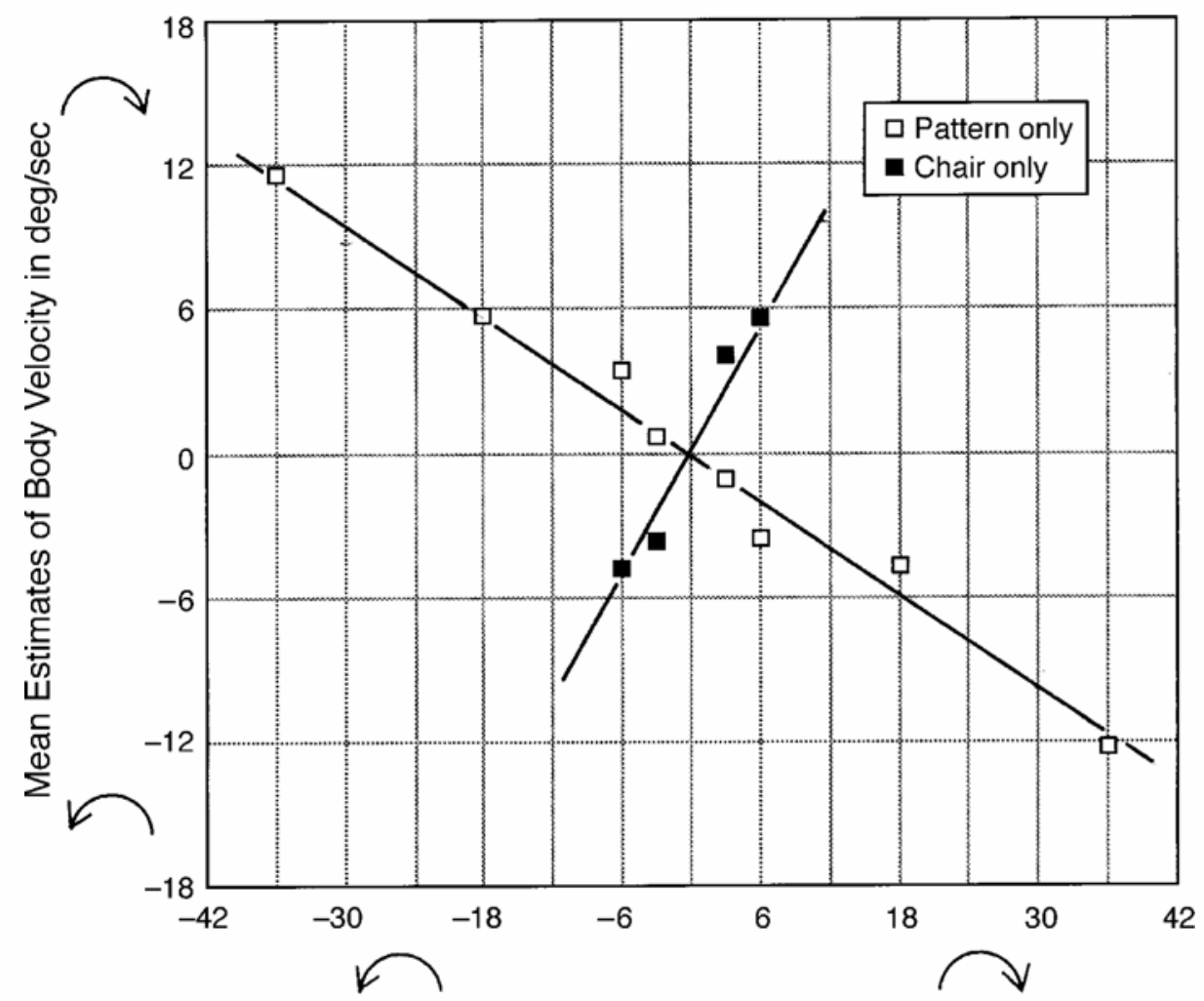

Pattern or Chair Velocity in deg/sec

Figure 12. Mean perceived velocity of self-motion that was produced by rotating the pattern alone (negative slope) and by rotating the chair alone (positive slope).

to the vection (i.e., the opposite condition), the perceived body motion was suppressed.

Huang and Young (1987) studied the human ability to control lateral body motion under several different visual field conditions. Each of their subjects was required to stabilize body sway by nullifying the lateral velocity of a sled on which the subject was seated. When the sled and the visual stimuli moved opposite to each other, the subjects succeeded best in stabilizing body sway; when the visual stimuli moved only to left or right, regardless of the sled motion, control of the sled was worst; when the visual stimuli moved concomitantly with the sled, control of the sled was moderate.

Carpenter-Smith et al. (1995) attempted to specify the relation between visual inputs and nonvisual inputs for the perception of self-motion. They demonstrated that a linear vection was nullified by a reverse passive motion of the subject and that there was an inverse relation between the velocity of a visual pattern and the backward body acceleration that was required to nullify the linear vection. Our study may support and supplement Carpenter-Smith et al.'s study, in that pattern velocity was traded for chair velocity over a wide range of perceived self-motion (Figures 6 and 10).

Much physiological evidence suggests that the activity of neurons in the vestibular nuclei is influenced by the motion of visual stimuli (for a review, see Dichgans \& Brandt, 1977; Howard, 1982). Although the exact physiological pathways serving visual-vestibular interactions are not fully known, the visual inputs may be assumed to reach the vestibular nuclei through the flocculus of the cerebellum, the inferior olive, and/or the pretectum in the midbrain. Our psychophysical data suggest that when a human body is rotated around a roll axis, perceived selfmotion is represented as a linear sum of the visual and the vestibulo-tactile components. We do not know whether such a simple linear relation holds for the neural activity of the vestibular nuclei, but we hope to provide a behavioral hypothesis that may be closely related to physiological findings in the future.

In conclusion, in two experiments, we replicated the findings of (1) a tradeoff of pattern velocity and chair velocity in maintaining constant velocity of perceived selfmotion and (2) a general dominance of pattern velocity in integrating pattern and chair velocities into perceived self-motion. Moreover, vestibulo-tactileinputs emanating from the chair motion were more effective for a body rolling slowly near the gravitational vertical than for a body rolling quickly with a large amplitude. From a broader perspective, our findings probably suggest that the combination of motion information from the peripheral sensory organs depends on the situation. Therefore, 
future work should focus on constructing a model that predicts how well visual and vestibulo-tactileinputs are combined at a higher perception of self-motion.

\section{REFERENCES}

Anderson, G. J. (1986). Perception of self-motion: Psychophysical and computational approaches. Psychological Bulletin, 99, 52-63.

Benson, J. A. (1990). Sensory functions and limitations of the vestibular system. In R. Warren \& A. Wertheim (Eds.), Perception and control of self-motion (pp. 145-170). Hillsdale, NJ: Erlbaum.

Berthoz, A., Pavard, B., \& Young, L. R. (1975). Perceptual linear horizontal self-motion induced by peripheral vision (linear vection): Basic characteristics and visual-vestibular interactions. Experimental Brain Research, 23, 471-489.

Brandt, T., Büchele, W., \& ARnold, F. (1977). Arthrokinetic nystagmus and ego-motion sensation. Experimental Brain Research, 30, 331-338.

Brandt, T., Dichgans, J., \& Koenig, E. (1973). Differential effects of central versus peripheral vision on egocentric and exocentric motion perception. Experimental Brain Research, 16, 476-491.

BRAndT, T., Wist, E. R., \& Dichgans, J. (1975). Foreground and background in dynamic spatial orientation. Perception \& Psychophysics, 17, 497-503.

Brown, J. H. (1966). Magnitude estimation of angular velocity during passive rotation. Journal of Experimental Psychology, 72, 169-172.

Carpenter-Smith, T. R., Futamura, R. G., \& Parker, D. E. (1995). Inertial acceleration as a measure of linear vection: An alternative to magnitude estimation. Perception \& Psychophysics, 57, 35-42.

Clark, B., \& Stewart, J. D. (1968). Magnitude estimates of rotational velocity during and following prolonged increasing, constant, and zero angular acceleration. Journal of Experimental Psychology, 78, 329-339.

DichGans, J., \& BRANDT, T. (1977). Visual-vestibular interaction: Effects on self motion perception and postural control. In R. Held, H. Leibowitz, \& H. L. Teuber (Eds.), Handbook of sensory physiology (Vol. VIII, pp. 755-804). Berlin: Springer-Verlag.

Dichgans, J., Held, R., Young, L. R., \& Brandt, T. (1972). Moving visual scenes influence the apparent direction of gravity. Science, 178, 1217-1219.

Dunker, K. (1929). Über induzierte Bewegung [On induced motion]. Psychologische Forschung, 12, 180-259. Translated and condensed in W. Ellis (Ed.), Source book of Gestalt psychology (pp. 161-172). New York: Humanities Press.

GiBson, J. J. (1966). The senses considered as perceptual systems. Boston: Houghton Mifflin.

Graybiel, A., \& Patterson, J. L. (1955). Thresholds of stimulation of the otolith organs as indicated by the oculogravic illusion. Journal of Applied Physiology, 7, 666-670.

Harris, L. R., Jenkin, M., \& Zikovitz, D. C. (2000). Visual and nonvisual cues in the perception of linear motion. Experimental Brain Research, 135, 12-21.

Held, R, Dichgans, J., \& Bauer, J. (1975). Characteristics of moving visual scenes influencing spatial orientation. Vision Research, 15, 357-365.

Howard, I. P. (1982). Human visual orientation. Chichester, U.K.: Wiley.

Howard, I. P., \& Childerson, L. (1994). The contribution of motion, the visual frame, and visual polarity of sensations of body tilt. Perception, 23, 753-762.

HuANG, J. K., \& Young, L. R. (1987). Influence of visual and motion cues on manual lateral stabilization. Aviation, Space, \& Environmental Medicine, 58, 1197-1204.

Kennedy, R. S., Hettinger, L. J., Harm, D. L., Ordy, J. M., \& Dunlap, W. P. (1996). Psychophysical scaling of circular vection (CV) produced by optokinetic (OKN) motion: Individual differences and effects of practice. Journal of Vestibular Research, 6, 331-341.

KLINKE, R, \& SCHMIDT, C. L. (1970). Efferent influence on the vestibular organ during active movements of the body. Pflügers Archiv für die gesamte Physiologie des Menschen und der Tiere, 318, 325-332.

Lackner, J. R., \& Graybiel, A. (1979). Parabolic flight: Loss of sense of orientation. Science, 206, 1105-1108.

Lackner, J. R. \& TeIXeIRA, R. A. (1977). Optokinetic motion sickness: Continuous head movements attenuate the visual induction of apparent self-rotation and symptoms of motion sickness. Aviation, Space, \& Environmental Medicine, 48, 248-253.

Lishman, J. R, \& LeE, D. N. (1973). The autonomy of visual kinaesthesis. Perception, 2, 287-294.

Melcher, G. A., \& HenN, V. (1981). The latency of circular vection during different accelerations of the optokinetic stimulus. Perception \& Psychophysics, 30, 552-556.

Mittelstaedt, H. (1999). The role of the otoliths in perception of the vertical and in path integration. In B. Cohen \& B. J. M. Hess (Eds.), Otolith function in spatial orientation and movement (Annals of the New York Academy of Sciences, Vol. 871, pp. 334-344). New York: New York Academy of Sciences.

Онмі, M. (1996). Egocentric perception through interaction among many sensory systems. Cognitive Brain Research, 5, 87-96.

Parker, D. E., Wood, D. L., Gulledge, W. L., \& Goodrich, R. L. (1979). Self-motion magnitude estimation during linear oscillation: Changes with head orientation and following fatigue. Aviation, Space, \& Environmental Medicine, 50, 1112-1121.

Quix, F. H. (1925). The function of the vestibular organ and the clinical examination of the otolithic apparatus. Journal of Laryngology \& Otology, 40, 425-443,493-511.

Reason, J. T., Mayes, A. R. \& Dewhurst, D. (1982). Evidence for a boundary effect in roll vection. Perception \& Psychophysics, 31, 139144.

SchöNe, H. (1964). On the role of gravity in human spatial orientation. Aerospace Medicine, 35, 764-772.

Schöne, H., \& Mortag, H.-G. (1968). Variation of the subjective vertical on the parallel swing at different body position. Psychologische Forschung, 32, 124-134.

SCHÖNE, H., \& WADE, N. J. (1971). The influence of force magnitude on the perception of body position: II. Effect of body posture. British Journal of Psychology, 62, 347-352.

TeiXeIRA, R. A., \& LACKNER, J. R. (1979). Optokinetic motion sickness: Attenuation of visually-induced apparent self-rotation by passive head movements. Aviation, Space, \& Environmental Medicine, 50, 264-266.

Udo DE HAEs, H. A. (1970). Stability of apparent vertical and ocular countertorsion as a function of lateral tilt. Perception \& Psychophysics, 8, 137-142.

van der Steen, F. A. M., \& Brockhoff, P. T. M. (2000). Induction and impairment of saturated yaw and surge vection. Perception \& Psychophysics, 62, 89-99.

Wong, S. C. P., \& Frost, B. J. (1978). Subjective motion and acceleration induced by the movement of the observer's entire visual field. Perception \& Psychophysics, 24, 115-120.

YounG, L. R. (1984). Perception of the body in space: Mechanisms. In J. M. Brookhart, V. B. Mountcastle, I. Darian-Smith, \& S. R. Geiger (Eds.), Handbook of physiology: Sect. 1. The nervous system: Vol. III. Sensory Processes: Pt. 2 (pp. 1023-1066). Bethesda, MD: American Physiological Society.

Young, L. R., Crites, T. A., \& Oman, C. M. (1983). Brief weightlessness and tactile cues influence visually induced roll. In C. R. Pfaltz (Ed.), Advances in oto-rhino-laryngology (Vol. 30, pp. 230-234). Basel: Karger.

Young, L. R, Dichgans, J., Murphy, R., \& Brandt, T. (1973). Interaction of optokinetic and vestibular stimuli in motion perception. Acta Oto-Laryngologica, 76, 24-31.

Young, L. R, Oman, C. M., \& Dichgans, J. M. (1975). Influence of head orientation on visually induced pitch and roll sensation. Aviation, Space, \& Environmental Medicine, 46, 264-268.

Young, L. R., Oman, C. M., Watt, D. G. D., Money, K. E., \& LichtenBERG, B. K. (1984). Spatial orientation in weightlessness and readaptation to earth's gravity. Science, 225, 205-208.

Young, L. R, \& Shelhamer, M. (1990). Weightlessness enhances the relative contribution of visually-induced self-motion. In R. Warren \& A. Wertheim (Eds.), Perception and control of self-motion (pp. 523538). Hillsdale, NJ: Erlbaum.

(Manuscript received April 25, 2001; revision accepted for publication January 5, 2002.) 